\title{
USO DA SUPLEMENTAÇÃO DA VITAMINA D DURANTE O PERÍODO GESTAÇÃO E LACTAÇÃO
}

\author{
USE OF VITAMIN D SUPPLEMENTATION DURING THE GESTATION \\ AND LACTATION PERIOD
}

\author{
Érica Vanessa Oliveira Gonçalves ${ }^{1}$ \\ Carolina Moreira de Santana ${ }^{2}$ \\ Bárbara Costa Paulino ${ }^{3}$ \\ Larissa Brito de Medeiros ${ }^{4}$
}

RESUMO: OBJETIVO: Verificar o uso da suplementação da Vitamina D durante a gestação, no puerpério, no período de lactação e nos lactentes. METODOLOGIA: Trata-se de uma pesquisa do tipo descritiva em delineamento transversal e com abordagem quantitativa. Participaram da pesquisa gestantes, lactantes e lactentes que atenderam aos critérios de inclusão e exclusão. Foi aplicado um questionário on-line com perguntas de múltipla escolha, elaborado para esse projeto, sobre o uso da suplementação da Vitamina D. A captação da amostra foi feita através da busca ativa com compartilhamento de informação via WhatsApp e Instagram. Os dados coletados foram analisados por meio do Microsoft Office Exce|® 2016, para realizar a análise mediante a estatística descritiva, sendo apresentados em gráficos. RESULTADOS: Das 45 participantes, $73 \%$ relataram que fora prescrita a suplementação de vitamina $D$ na gestação, enquanto $27 \%$ não foram prescritas. Para lactantes, $71 \%$ afirmaram que utilizaram e $29 \%$ não utilizaram a suplementação da vitamina. Já em bebês, $73 \%$ relataram que houve a prescrição e $27 \%$ relataram que não foi prescrito. Quando questionadas se fizeram o uso da suplementação da vitamina D na gestação, $30(66,7 \%)$ afirmaram que fizeram o uso, $14(31,1 \%)$ não realizaram o uso e apenas $1(2,2 \%)$ não recorda se fez o uso durante a gestação. Quando interrogadas se ofertam a suplementação aos seus bebês, $32(71,1 \%)$ declararam fornecer e $13(28,9 \%)$ não ofertaram. Para esse quesito, foram excluídas $6(13,3 \%)$ participantes, pois relataram ainda estar gestantes. CONCLUSÃO: Notouse que muitas mães sabem sobre a importância da VD para a sua gestação, bem como para lactação e, também para os seus filhos. Além disso, foi verificado que ocorreu prescrição da vitamina na gestação e para os bebês na maioria das

\footnotetext{
${ }^{1}$ Graduanda em Nutrição da Faculdade Santa Maria - FSM.

${ }^{2}$ Mestre em Sistemas Agroindustriais. Professora da Faculdade Santa Maria - FSM.

${ }^{3}$ Mestre em Nutrição. Professora da Faculdade Santa Maria - FSM.

${ }^{4}$ Doutora em Nutrição. Professora da Faculdade Santa Maria - FSM.
} 
participantes de acordo com suas necessidades. Já nos bebês, o estudo mostrou que a maioria faz o uso da suplementação de acordo com a necessidade de cada um. Além disso, a pesquisa é de grande relevância nessa área, tornando-se importante para futuros estudos, nele possibilitou verificar, que mesmo em meio à grande veiculação dos benefícios da vitamina $\mathrm{D}$ para o organismo, pode-se observar que ainda não existe uma recomendação exata de suplementação para as gestantes e lactantes.

Palavras-chave: Vitamina D. Suplementação. Gestante. Lactante. Lactente.

ABSTRACT: OBJECTIVE: To verify the use of Vitamin D supplementation during the pregnancy, puerperium, in the lactation period and in infants. METHODOLOGY: This is a research of descriptive type in cross delineation and with quantitative approach. Attended the research pregnant woman, breastfeeding women and breastfeeding women fulfill inclusion and exclusion requirements. It was apply an online questionnaire with questions of multiple choice elaborate for this Project, about the use of Vitamin $D$ supplementation. The sample capture was make through active search with information sharing by WhatsApp and Instagram. The data collected were analyzed by means of Microsoft Office 2016, to realize the analysis through descriptive statistics, being presented in graphics. RESULTS: Of the 45 participants, $73 \%$ reported that vitamin $D$ supplementation was prescribed during pregnancy, while $27 \%$ were not prescribed. For breastfeeding women, $71 \%$ assert that used and $29 \%$ didn't use supplementation of vitamin. In babies, $73 \%$ reported that there was a prescription and $27 \%$ reporte that it wasn't prescribed. When questionaded if they used Vitamin D of suppplementation in pregnancy, 30 (66,7\%) assert that used, 14 $(31,1 \%)$ din't use and Only 1 (2,2\%) didn't remember if made the use during the pregnancy. When interviewed if they offer supplementation to their babies, 32 $(71,1 \%)$ declared to offer and 13 (28,9\%) didn't offer. For this aspect, were excluded $6(13,3 \%)$ participants, because They still reported being pregnant. CONCLUSION: It was noticed that many mothers knows about the importance of Vitamin $D$ for their pregnancy, as well as lactation and, also to their children. In addition, it was verified that vitamin occured during pregnancy and for babies in most participants in agreement with their needs. In babies, the study showed the most participants that made use supplementation in agreement with their needs each one. Moreover, the study is of great importance in this area, become importante for the future studies, in which it was possible to verify, that even in the midst of the great dissemination of the benefits of Vitamin $D$ for the organism, it can be observed that there is still no exact recommendation of supplementation for pregnant and breastfeeding women.

Keywords: Vitamin D. Supplementation. Pregnant Woman. Lactating. Infant. 


\section{INTRODUÇÃO}

A vitamina D (VD), é uma vitamina lipossolúvel de grande importância materno-infantil, ajudando na mobilização do cálcio no osso, na absorção do cálcio, na absorção do fosfato no intestino, além de contribuir para o sistema imunológico. Com isso, sua deficiência e suplementação vem sendo um dos assuntos mais pesquisados nessa área (HOLICK, 2007; PAULA et al., 2021).

As principais formas de sua obtenção são através da suplementação, da exposição solar e uma ingestão alimentar adequada do micronutriente. Essa vitamina é encontrada fisiologicamente em duas formas nos alimentos: a Vitamina D3 (colecalciferol), encontrada em alimentos de origem animal (gema de ovo, leite materno, fígado de boi, fórmulas lácteas fortificadas) e a Vitamina D2 (ergocalciferol), encontrada em alimentos de origem vegetal, obtidos por algumas plantas e fungos (HOLICK, 2007).

A hipovitaminose $D$ ou deficiência da vitamina $D(D V D)$, é relacionado com a falta ou a baixa absorção desse micronutriente. Diante disso, a hipovitaminose $D$ ou DVD, é caracterizado como um problema frequente e global, atingindo todas as faixas etárias, inclusive, gestante e crianças devido a vários fatores, como: a baixa exposição solar, pouca ingestão da vitamina $\mathrm{D}$ e até mesmo fatores fisiológicos referente a obesidade, gravidez, crianças obesas, síndromes de má absorção (PREMAOR; FURLANETTO, 2006; PAULA et al., 2021).

É elevado o número de gestantes que apresentam DVD de uma forma mais grave, o que proporciona maiores riscos durante a gestação, estando relacionada a complicações como pré-eclâmpsia, diabetes mellitus gestacional, depressão antenatal e puerpério e a prematuridade (MULLIGAN et al., 2010; PAULA et al., 2021). Esse risco elevado pode aparecer tanto na gestação, quanto no puerpério, como também no pós-parto. O reflexo dessa deficiência no lactente tende a ser mais grave, com consequências para toda a vida, gerando deficiências ósseas, como baixo peso ao nascer, osteomalácia, raquitismo infantil, fraturas frequentes, atraso 
no crescimento e desenvolvimento motor, irritabilidade, sudorese e atraso na erupção dentária (QUERIDO et al., 2017; NIGRI et al., 2019).

Atualmente o prognóstico da gestação é influenciado pelo estado nutricional materno antes e durante a gestação, obtendo um grande impacto sobre o crescimento e o desenvolvimento do $\mathrm{RN}$, pois o fornecimento desse micronutriente para o feto, depende exclusivamente da mãe. Com isso, a vitamina $D$ desempenha um papel em todas as fases da gravidez (GOULART; GOULART, 2017).

Nas primeiras semanas de vida, os níveis da VD são dependentes do que foi adquirido via placenta durante a vida uterina. Depois da oitava semana, os lactentes amamentados exclusivamente têm risco elevado de desenvolver hipovitaminose $D$, pois o leite humano tem baixa concentração de VD (aproximadamente 20 - 60 IU/L; 1,5 - 3\% do nível materno). Essa concentração não é suficiente para manter níveis ótimos de VD, sobretudo quando a exposição solar é limitada (CANADIAN PAEDIATRIC SOCIETY, 2007; URRUTIA-PEREIRA; SOLE, 2015).

Não há indicação da suplementação geral de vitamina $\mathrm{D}$ para toda a população. Para pessoas consideradas de risco, como mulheres grávidas e amamentando, com isso, para manter o valor de referência da vitamina $D$ definido por Sociedade Brasileira de Patologia Clínica - Medicina Laboratorial (SBPC/ML) e Sociedade Brasileira de Endocrinologia e Metabologia (SBEM) 1.000 UI de vitamina D por dia para manter os níveis de 30 a 60 ng / ml, mas não há consenso sobre esta recomendação até aqui (POMPEI; STEINER, 2019).

A Sociedade Brasileira de Pediatria (SBP), a Academia Americana de Pediatria (AAP) e a Recomendações do Consenso Global Sobre Prevenção e Manejo do Raquitismo Nutricional, indicam que a suplementação de vitamina D imediatamente após o nascimento, sendo adequado para todas as crianças de 0 12 meses, a utilização da dose diária de 400Ul/dia, independente da sua alimentação. Ja para bebês prematuros, é recomendado a suplementação de $400 U \mathrm{Ul} / \mathrm{dia}$, quando o peso é maior que $1.500 \mathrm{~g}$ e ter tolerância a ingestão oral. Já naqueles de 12 - 24 meses, a Sociedade Brasileira de Pediatria (SBP), faz a recomendação da suplementação de 600Ul/dia (NIGRI et al., 2019). 
Diante do exposto, esse trabalho tem como objetivo observar quanto a suplementação profilática da vitamina $D$ na gestação, no puerpério e entre os lactentes, além de identificar o uso e dosagem da suplementação da VD.

\section{METODOLOGIA}

Trata-se de uma pesquisa do tipo descritivo com delineamento transversal e abordagem quantitativa. Participaram da pesquisa mulheres que atenderem aos critérios de inclusão e exclusão.

Os critérios de inclusão foram, gestantes com gestação única ou gemelar, lactentes e mulheres que deram à luz e a criança que tenha até 12 meses, ser alfabetizada, usuário das redes sociais e que responderam corretamente o formulário on-line. Os critérios de exclusão foram, mães com crianças maiores de 1 ano e aquelas que não concordarão em participar da pesquisa.

O projeto foi submetido ao Comitê de Ética e Pesquisa (CEP) da Faculdade Santa Maria (FSM), por intermédio da Plataforma Brasil e logo após sua aprovação com o número do Parecer: 5.084.420 (ANEXO 1), foi realizada da coleta de dados.

A coleta foi realizada no mês de novembro de 2021. A ferramenta utilizada foi - Google Forms, considerado uma ferramenta de pesquisa institucional atual (MATHIAS; SAKAI, 2018). O questionário on-line de múltipla escolha com 15 questões abordou sobre o uso da suplementação da Vitamina $D$ e sua posologia. $O$ preenchimento só aconteceu após a aceitação do TCLE, caso a participante não concordasse com os termos, a pesquisa foi automaticamente direcionada para o fim do formulário. Os dados coletados foram analisados por meio do Microsoft Office Excel® 2016, para realizar a análise através de estatística descritiva, sendo apresentados em gráficos. 


\section{RESULTADOS E DISCUSSÃO}

A pesquisa contou com a participação de 45 mulheres, mães com idades entre 19 anos e 43 anos, tendo média de $\pm 28,82$ anos, destas 48,9\% ( $N$ 22) considera-se branca, 44,4\% (N 20) admite-se parda/mulata e 6,7\% (N 3) declara-se negra. Além de observar que 62,2\% (N 28) das participantes são casadas, 22,2\% ( $N$ 10) em união estável e $15,6 \%$ (N 7) solteira.

Em relação ao grau de escolaridade, $33,3 \%$ (N 15) possui pós-graduação completa, 6,7\% (N 3) pós-graduação incompleta, 22,2\% (N 10)) ensino superior completo, $20 \%(\mathrm{~N}$ 9) ensino superior incompleto, $13,3 \%(\mathrm{~N} 6)$ ensino médio completo, 2,2\% (N 1) ensino médio incompleto e 2,2\% ( $N$ 1) fundamental completo. Considerando os dados das 45 participantes obtidas, analisou-se que a renda familiar dessas mulheres, são 46,7\% (N 21) maior que três salários mínimos, 11,1\% (N 5) de dois e meio até 3 salários mínimos, 17,8\% (N 8) de um e meio até dois e meio salário mínimo, 13,3\% (N 6) de um até um e meio salário mínimo, 6,7\% (N 3) de meio até um salário mínimo e 4,4\% (N 2) até meio salário mínimo.

Quando perguntado sobre a gestação 68,9\% (N 31) das participantes responderam que estão na $1^{\underline{a}}$ gestação, $26,7 \%\left(N\right.$ 12) na $2^{\underline{a}}$ e $4,4 \%\left(N\right.$ 2) na $3^{\text {a }}$ gestação. Ao indagar sobre a prescrição da suplementação de vitamina D (VD) na gestação, $73 \%$ ( $N$ 33) das entrevistadas informaram que durante a gestação foi prescrição o uso da suplementação, já as $27 \%$ ( $N$ 12) informaram que não foi prescrito (Gráfico 1). Sobre a importância da vitamina D durante a gestação, $82 \%$ sabem da importância na gestação e apenas 18\% não sabem. 
GRÁFICO 1 - Prescrição da Suplementação de Vitamina D na Gestação.

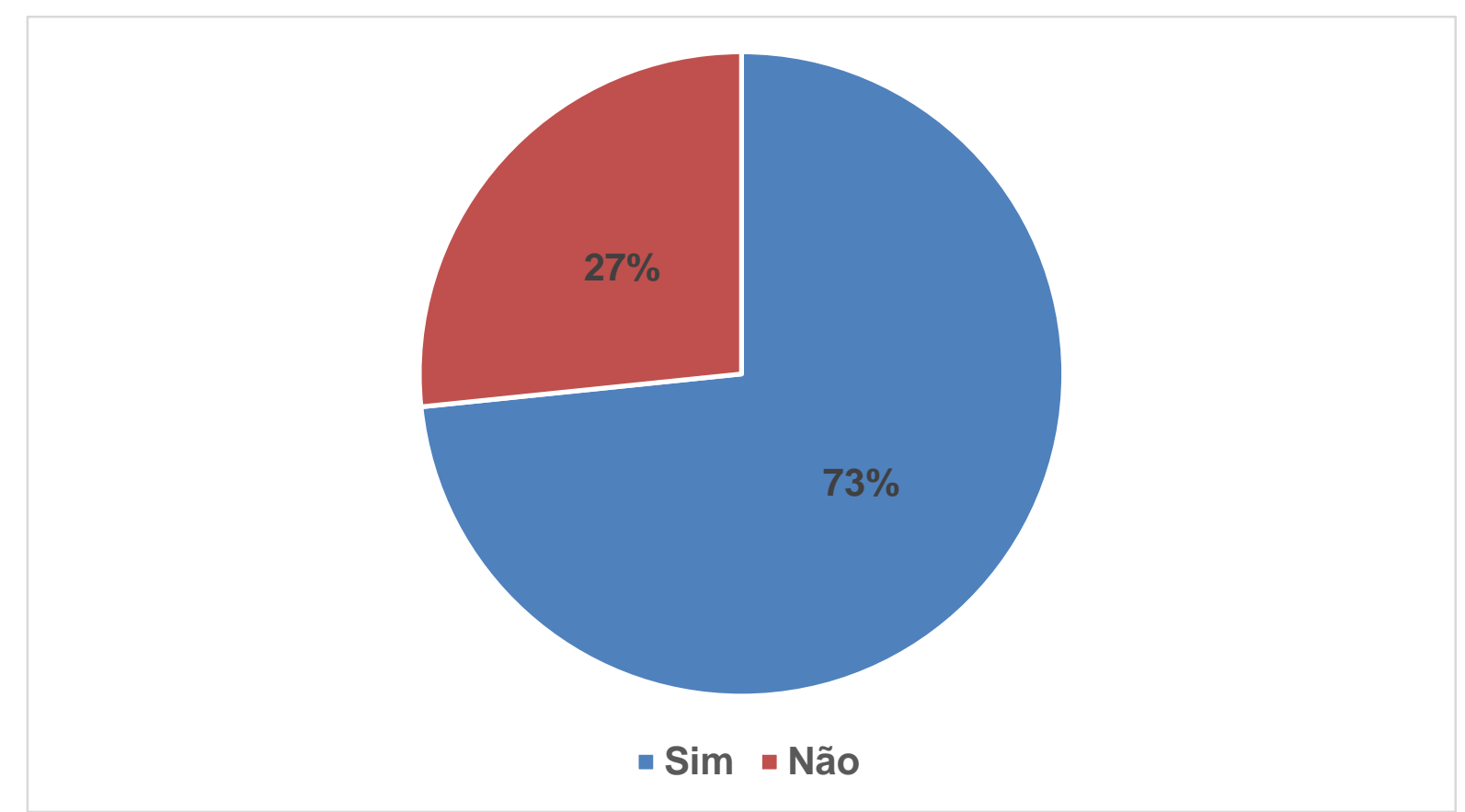

Fonte: Próprio autor (2021).

De-REGIL et al. (2016), em seu estudo sobre o uso generalizado de polivitamínicos pré-natais, apresentou que existe uma alta prevalência de diversas complicações associadas à hipovitaminose $D$ durante a gestação. Porém devem ser abordadas algumas estratégias de prevenção, assegurando a vitamina $D$ durante a gestação a fim de diminuir o impacto na saúde do recém-nascido (NETO, 2017; URRUTIA - PEREIRA; SOLÉ, 2015). No estudo de El Rifai e colaboradores (2013), afirma que os níveis maternos de vitamina $D$ se correlacionam fortemente com os níveis neonatais.

Alguns estudos têm demostrado que mulheres durante a gestação, tem os níveis de vitamina $D$ intimamente relacionado com a concentração desse hormônio no cordão umbilical, e a falta desse hormônio durante o período fetal pode ter efeitos permanentes na fisiologia e no metabolismo do recém-nascido (GOULART; GOULART, 2017).

A vitamina $D$ interfere em muitos mecanismos que estão envolvidos na patogênese da pré-eclâmpsia, na regulação imunológica, no controle da pressão 
arterial, diabetes mellitus gestacional e parto prematuro pode estar relacionado à Hipovitaminose D (BENACHI et al., 2013).

Basile (2014) relata que além dos efeitos diretos, alguns danos causados pela Deficiência da Vitamina D (DVD) podem se manifestar depois de muitos anos, portanto, a suplementação é essencial na gestação. A suplementação de vitamina $D$ durante a gestação tem sido recomendada como uma intervenção para prevenir esses resultados adversos ao bebê.

No gráfico 2, mostra que quando abordadas se fizeram 0 uso da suplementação da vitamina D durante a gestação, 30 entrevistadas (66,7\%) afirmaram que fizeram o uso, 14 delas (31,1\%) não suplementaram e apenas 1 entrevistada $(2,2 \%)$ não recorda se fez o uso durante a gestação.

GRÁFICO 2 - Uso da Suplementação da Vitamina D Durante a Gestação.

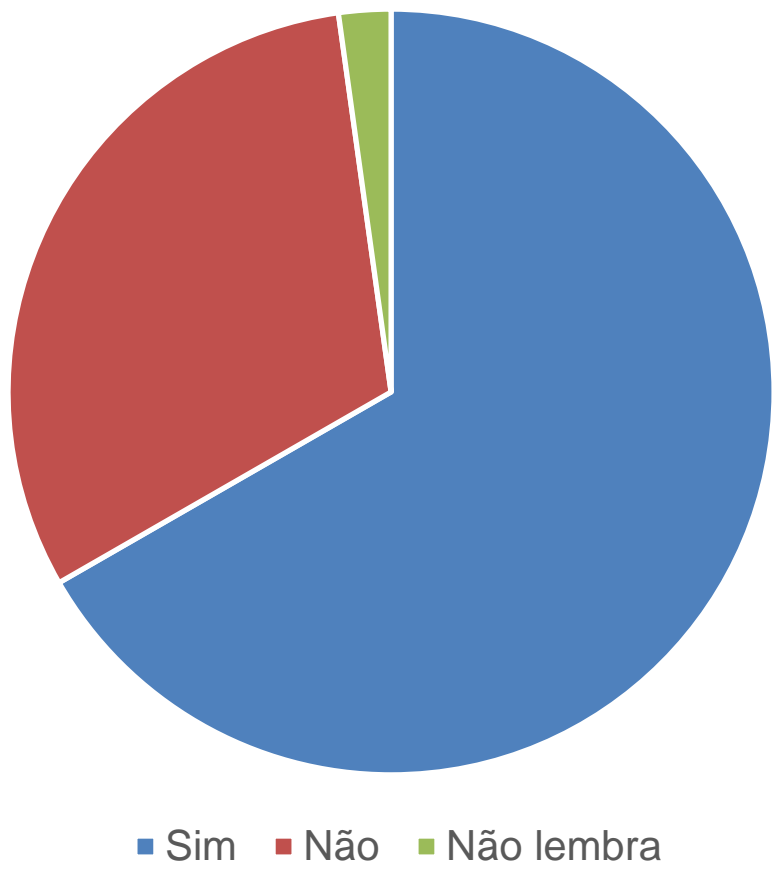

Fonte: Próprio autor (2021).

Sobre a posologia da suplementação de VD, 27 participantes (59,96\%) informaram as seguintes dosagens: 2,22\% (N 1) utilizou a dosagem de $20.000 \mathrm{UI}$; 2,22\% (N 1) fez uso de 15.000 Ul; 4,44\% (N 2) suplementaram com 10.000 UI, já $11,11 \%$ (N 5) responderam que utilizaram de $7.000 \mathrm{UI} ; 8,88 \%$ (N 4) administraram a 
dosagem de $5.000 \mathrm{Ul}$; outras 4,44\% (N 2) usaram de $4.000 \mathrm{UI}$; 2,22\% (N 1) utilizou a dose de $3.000 \mathrm{UI}$; 19,99\% (N 9) receberam a quantidade de $2.000 \mathrm{UI}$ e 4,44\% (N 2) fizeram uso de $1.000 \mathrm{UI}$ de vitamina $\mathrm{D}$.

O restante da amostra, 14 participantes $(31,08 \%)$ afirmaram que não foi necessário o uso da suplementação, 3 participantes $(6,66 \%)$ fizeram o uso, porém não recordavam a dosagem utilizada e 1 participante (2,22\%) alegou que 0 recomendado teria sido apenas o banho de sol nas mamas pela manhã durante a gestação.

Segundo a recomendação do Ministério da Saúde, os seios devem ser preparados para o aleitamento materno ainda na gestação, com isso, os profissionais de saúde durante o pré-natal devem orientar a mãe em expor as mamas ao sol, pelo menos 1x ao dia, preferencialmente pela manhã (SILVA et al., 2014).

Benachi et al. (2013), diz em seu estudo que a suplementação de vitamina D durante a gestação é controversa e que algumas sociedades consideram desnecessário, enquanto outras sugerem até $2.000 \mathrm{UI} / \mathrm{dia}$. Outra pesquisa recente provou a segurança e eficácia em tomar doses mais altas quanto a de $4.000 \mathrm{UI} / \mathrm{dia}$ de vitamina $D$ durante a gestação (NETO, 2017). Não existe quantidade precisa ou um ponto de corte que defina essa suplementação, ou seja, cabe ao profissional obstetra ou nutricionista e à necessidade de suplementação da gestante.

Nos últimos meses de gestação há um aumento na transferência de vitamina D da mãe para o feto por meio da placenta. Esta é a primeira fonte de obtenção de VD do recém-nascido (CAMARGO et al., 2011). Depois do nascimento, o bebê ncessita de um suporte nutricional e o leite materno é o alimento nutricionalmente completo, porém a concentração de vitamina $D$ é insuficiente (FERREIRA, 2003).

De acordo com Dawodu e Akinbi (2013), nas primeiras semanas de vida, os níveis de vitamina $D$ depedem das substâncias obtidas através da vida placentária. Após a oitava semana de vida, a VD é reduzida de modo que os lactentes amamentados exclusivamente pelo leite materno obtêm maior risco de desenvolver DVD, porque o teor de VD no leite materno é baixo e insuficiente para manter níveis adequados. Com destaque especialmente nos casos onde a luz do sol se faz limitada. 
No gráfico 3 apresenta os resultados referente a utilização de vitamina $D$ durante o aleitamento materno, o resultado foi que $71 \%$ ( $N$ 32) da amostra suplementaram durante o aleitamento materno e $29 \%$ ( $N$ 13) não utilizou.

GRÁFICO 3 - Utilização de vitamina D durante o aleitamento materno.

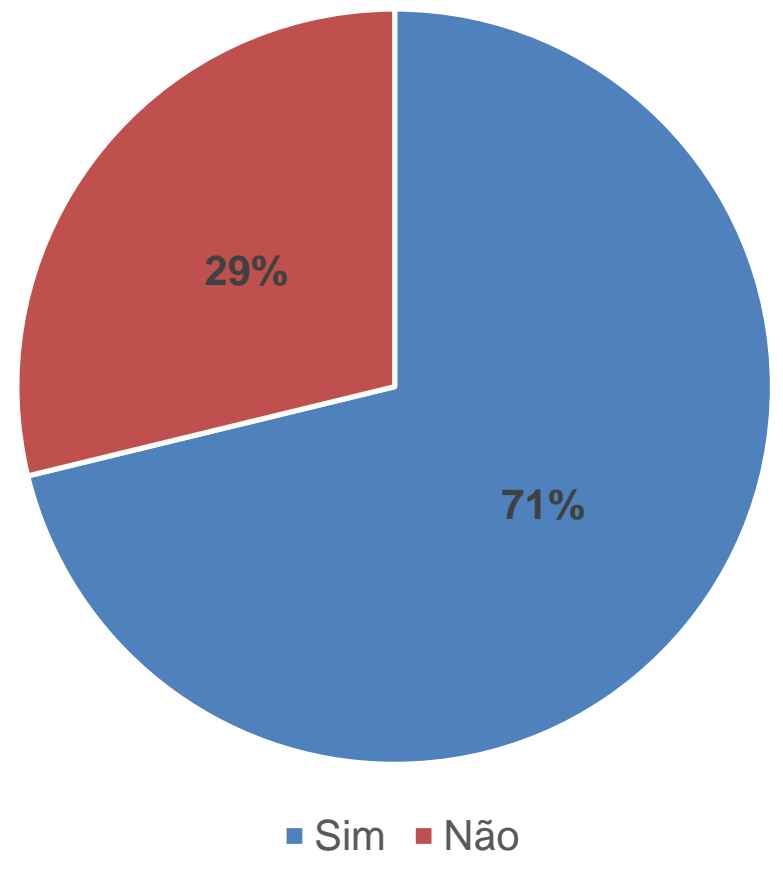

Fonte: Próprio autor (2021).

Os autores Pompei e Steiner (2019) afirmam que pessoas consideradas de risco, como gestantes e lactantes, para que mantenham o valor de referência de vitamina D definido pela Sociedade Brasileira de Patologia Clínica - Medicina Laboratorial (SBPC/ML) e Sociedade Brasileira de Endocrinologia e Metabologia (SBEM), é recomendado a suplementação de $1000 \mathrm{UI} / \mathrm{d}$ para manter os níveis adequados de VD, mas atualmente não há consenso sobre essa recomendação.

Para a Sociedade Brasileira de Pediatria (2014), são considerados grupos de risco para a deficiência de vitamina D: crianças amamentadas ao seio sem suplementação, sem exposição solar adequada e/ou limitada, com sindrome de máabsorção intestinal e com má-absorção de gorduras.

Diante disso, o gráfico 4 apresenta sobre a prescrição da suplementação de vitamina $D$ para o bebê. Para esse quesito foram excluídas 6 participantes $(13,3 \%)$, 
pois relataram ainda estar gestantes, com isso, é possivel observar que 73\% (N 32) foi prescrito a suplementação e $27 \%$ (N 13) afirmaram que não foi prescrito a VD.

GRÁFICO 4 - Prescrição da suplementação da vitamina D em bebê.

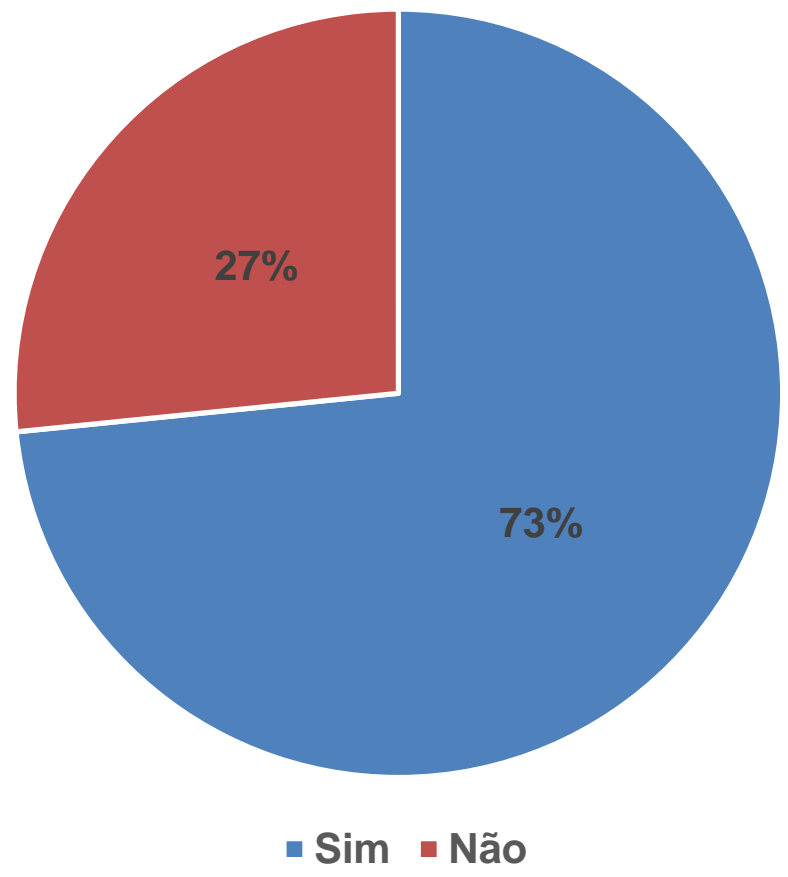

Fonte: Próprio autor (2021).

Brasil (2014) diz que o lactente recebe aleitamento materno exclusivo conforme a necessidade e é regularmente exposta ao sol, ou se recebe pelo menos $500 \mathrm{~mL} /$ dia de fórmula infantil, a suplementação de vitamina $D$ não é necessária. Porém, recomenda-se suplementar quando a exposição solar for insuficiente e para prevenção de doenças ósseas futuras.

Quando interrogadas se ofertam a suplementação aos seus bebês, 32 participantes $(71,1 \%)$ declararam que fizeram ou fazem uso da suplementação e 13 participantes (28,9\%) não ofertaram a suplementação de VD. Logo em seguida, foram questionadas sobre a importância da vitamina $D$ para o bebê e das 45 participantes, 39 (86,7\%) afirmaram saber da importância e 6 (13,3\%) disseram não saber.

Para D'Amelio e Quacquerelli (2020), a suplementação de vitamina D em lactentes torna-se necessário em casos de prevenção de algumas condições 
clínicas, como: fraqueza muscular, sinais e sintomas de raquitismo ou osteomalácia, fosfatase alcalina elevada, sinais radiológicos sugerindo osteopenia.

Posto isso, a exposição à vitamina $\mathrm{D}$ de modo inadequado durante $\mathrm{O}$ desenvolvimento fetal, afeta a regulação do sistema imunológico do recém-nascido, incluindo medidas do tamanho fetal, composição corporal, além de complicações na infância, como problemas respiratórios nos primeiros anos de vida (HORNSBY et al., 2018; CURTIS et al., 2018).

Para garantir um suprimento adequado dessa vitamina e evitar sua deficiência, a Academia Brasileira de Pediatria (2014), recomenda a suplementação de forma preventiva de $400 \mathrm{UI} /$ dia desde a primeira semana de vida até os 12 meses. Além disso, recomenda $600 \mathrm{Ul} / \mathrm{dia}$ de 12 a 24 meses, incluindo crianças exclusivamente amamentadas, independente da região do país (SBP, 2014).

A duração da suplementação de vitamina $D$ ainda não foi determinada e é recomendado que crianças com fatores de risco a suplementem. Já as crianças saudáveis, sem fatores de risco, mas com exposição solar limitadas, devem ser suplementadas e avaliadas separadamente (NIGRI, 2019).

\section{CONCLUSÃO}

Notou-se que muitas mães sabem sobre a importância da VD para a sua gestação, para lactação, assim como também para os seus filhos. Além disso, foi verificado que ocorreu prescrição da vitamina à maioria das participantes de acordo com suas necessidades durante o período gestacional, como para os bebês. Nela constatou, que muitas fizeram o uso da suplementação por saberem da importância na gestação, porém algumas delas, mesmo com a prescrição, resolveram não fazer o uso. Outras relataram que não foi necessário, pois já atingiam os níveis necessários na gestação. Já nos bebês, o estudo mostrou que a maioria faz o uso da suplementação de acordo com a necessidade de cada um.

Outro ponto encontrado no estudo, foi que existem recomendações da Sociedade Brasileira de Pediatria para suplementação profilática de vitamina $D$ em 
bebês, no intuito de evitar doenças ósseas futuras e suprir as necessidades, quando não há exposição à luz solar adequadamente para o bebê. Ademais, o estudo é de grande relevância nessa área, tornando-se importante para futuras pesquisas, nele possibilitou verificar que, mesmo em meio à grande veiculação dos benefícios da vitamina $D$ para o organismo, pode-se observar que ainda não existe uma recomendação exata de suplementação para as gestantes e lactantes.

\section{REFERÊNCIAS BIBLIOGRÁFICAS}

BASILIE, L. H. Gestante e necessidade da vitamina D. International Journal of Nutrology, v.7, n.1, p. 05-13, 2014.

BENACHI, A.; CORDIER, A.G.; COURBEBAISSE, M.; SOUBERBIELLE, J.C. Vitamin D and pregnancy. Presse Med., v. 42, n. 10, p. 1377-1382, 2013.

CAMARGO, C. A. et al. Cord-blood 25-hydroxyvitamin D levels and risk of respiratory infection, wheezing, and asthma. Pediatrics, Springfield, v. 127, n.1, p. 2010-2442. 2011.

CANADIAN PAEDIATRIC SOCIETY. Vitamin D supplementation: recommendations for Canadian mothers and infants. Paediatr Child Health, v. 12, p. 583-9, 2007.

CURTIS, E. M. et al. Maternal vitamin D supplementation during pregnancy. Br Med Bull, v. 126, n. 1, p. $57-77,2018$.

D'AMELIO, P; QUACQUARELLI, L. Hypovitaminosis D and Aging: $s$ There a Role in Muscle and Brain Health? Nutrients, v. 12, n. 3, p. 628, 2020.

DAWODU, A.; AKINBI, H. Nutrição com vitamina D na gravidez: opinião atual. Int $J$ Womens Health, v. 5, p.333-343, 2013.

De-REGIL, L. M. et al. Vitamin D supplementation for women during pregnancy (review). Cochrane Database Syst Ver, v. 1, 2016.

E HORNSBY, E. et al. Vitamin D supplementation during pregnancy: Effect on the neonatal imune system in a randomized controlled trial. J Allergy Clin Immunol, v. 141, n. 1, p. 269-278, 2018.

EL RIFAI, N.M.; ABDEL, M.G.A.; GAAFAR, H.M.; HAMED, D.A. Vitamin D deficiency in Egyptian mothers and their neonates and possible related factors. J Matern Fetal Neonatal Med, v. 27; n. 10; p. 1064 -1068. Julho de 2014. Doi: 10.3109 / 14767058.2013.849240.

FERREIRA, M; CASCAIS, C.S. Novas Recomendações para a Suplementação de Vitamina D. Rev Port Clin Geral, v19, n. 4, p. 292, 2003.

GOULART, P. A. M.; GOULART, R. N. Gestação e Deficiência de Vitamina D: Artigo de Revisão na Literatura. Arquivos Catarinenses de Medicina, v. 46, n. 1, p. 173-181, 2017.

HOLICK, M. F.; CHEN T. C. Vitamin D deficiency: a worldwide problem with health consequences. Am J Clin Nutr, v. 87, n. 4, 2008. 
MATHIAS, S. L.; SAKAI, C. Utilização da ferramenta google forms no processo de avaliação institucional: estudo de caso nas faculdades Magsul. Rev. Interfaces, v. 5, n. 15, p. 71-77, 2018.

MULLIGAN, M. L. et al. Implications of vitamin D deficiency in pregnancy and lactation. American journal of obstetrics and gynecology, v. 202, n. 5, p. 1-9, 2010.

NETO, M. C. Papel da vitamina D na gestação. In: A importância da vitamina $D$ na saúde da mulher. São Paulo: Federação Brasileira das Associações de Ginecologia e Obstetrícia; 2017. Cap. 4, p.28-37. (Série Orientações e Recomendações FEBRASGO; no.14/Comissão Nacional Especializada em Osteoporose).

NIGRI, A. A. et al. Vitamina D quando fazer a dosagem e tartar? Pediatra Atualize-se Boletim da Sociedade de Pediatria de São Paulo, ano 4, n. 5, 2019.

PAULA, L. C. et al. Implicações da deficiência materna de vitamina D: uma revisão sistemática. FEMINA, v. 49, n.1, p. 44-51, 2021.

POMPEI, L. de M.; STEINER, M. L. Vitamina D. Além da nutrição - O impacto da nutrição materna na saúde das futuras gerações. $1^{\circ}$ Ed. p. 97-106, 2019.

PREMAO, M. O.; FURLANETTO, T. W. Hipovitaminose D em adultos: entendendo melhor a apresentação de uma velha doença. Arq Bras Endocrinol Metab, v. 50, n. 1, 2006.

PRODANOV, C. C.; FREITAS, E. C. Metodologia do Trabalho Científico: Métodos do Trabalho da Pesquisa e do Trabalho Acadêmico. 2 ed. Novo Hamburgo: Feevale, 2013.

QUERIDO, A. C. C. M. et al. Implicações maternas e fetais da hipovitaminose D no período gestacional: uma revisão de literatura. Revista de Patologia do Tocantins, v. 4, n. 4, p. 73-80, 2017.

SBP - Sociedade Brasileira de Pediatria. Deficiência de vitamina D em crianças e adolescentes. Documento Científico, 2014.

Departamento Científico de Endocrinologia: SBP. Hipovitaminose $\mathbf{D}$ em pediatria: recomendações para o diagnóstico, tratamento e prevenção. Guia Prático de Atualização, n. 1, 2016.

SILVA, S. R., et al. Práticas de autocuidado desarrolhadas por gestantes atendidas em ambulatório de pré-natal. Rev. Eletr. Enf. [Internet]. v.16, n.4, p. 812 - 821, 2014.

URRUTIA-PEREIRA, M.; SOLE, D. Deficiência de vitamina $D$ na gravidez e o seu impacto sobre o feto, o recém-nascido e na infância. Rev. paul. pediatr., v. 33, n. 1, p. 104-113, 2015. 


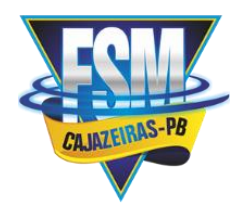

ANEXO 1 - PARECER DA PLATAFORMA BRASIL

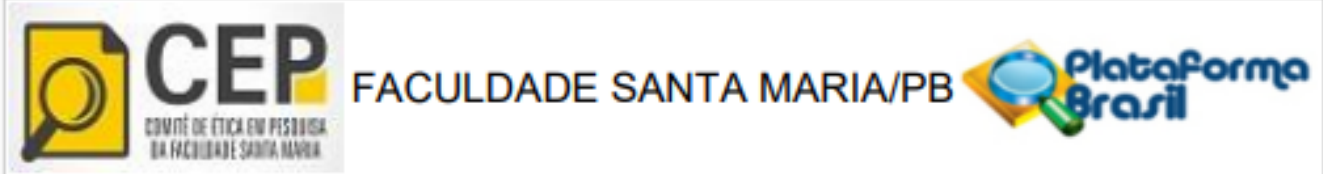

\section{PARECER CONSUBSTANCIADO DO CEP}

\section{DADOS DO PROJETO DE PESQUISA}

Titulo da Pesquisa: USO DA SUPLEMENTAÇĀO DA VITAMINA D DURANTE O PERIODO DA GESTAÇĀO E LACTAÇẢO

Pesquisador: CAROLINA MOREIRA DE SANTANA

Área Temática:

Versăo: 5

CAAE: 51730421.3 .0000 .5180

Instituiçăo Proponente: Faculdade Santa Maria/ FSM /PB

Patrocinador Principal: Financiamento Próprio

\section{DADOS DO PARECER}

Número do Parecer: 5.084 .420

\section{Apresentaçăo do Projeto:}

A vitamina D (VD), é uma vitamina lipossolúvel de grande importância materno-infantil, ajudando na mobilização do cálcio no osso, além de contribuir para o sistema imunológico. Sua deficiência ou hipovitaminose, é relacionado com a falta ou a baixa absorção desse micronutriente. É alta a prevalência de gestantes que apresentam deficiência de vitamina D (DVD) de uma forma mais grave, proporcionando mais riscos na gestação, puerpério e pós-parto. Além de estar relacionada a complicaçōes como pré-eclåmpsia, diabetes mellitus gestacional, depressão antenatal e puerpério e a prematuridade. Para pessoas consideradas de risco, como mulheres grávidas e amamentando, não há consenso sobre esta recomendação até aqui. Diante disso, a suplementaçăo profilática oral de vitamina $\mathrm{D}$ na infância deverá ser realizada na dose de $400 \mathrm{UI} /$ dia, a partir das primeiras semanas de vida até os 12 meses e de $600 \mathrm{UI} /$ dia dos 12 aos 24 meses de vida de modo inclusivo para crianças em aleitamento materno exclusivo, assim garantindo a prevenção deficiências nutricionais com potencial agravante na saúde materno-infantil. OBJETIVO: Observar o uso da suplementação da Vitamina D durante a gestação, no puerpério e no periodo de lactação. METODOLOGIA: Trata-se de uma pesquisa do tipo descritivo em delineamento transversal e com abordagem quantitativa. A pesquisa terá como público alvo gestante, lactantes e bebês, no qual será conduzida de forma aleatória, de senso, com todos os individuos que atenderem aos critérios de inclusão. Será aplicado um questionário online com perguntas de 